\section{How do bacteria acquire plant genes?}

SIR-Carlson and $\mathrm{Chelm}^{1}$ have presented evidence of gene transfer from a plant to a closely associated bacterial symbiont of the family Rhizobiaceae. But one particular feature of the purportedly transferred glnII gene, namely the lack of introns, suggests an alternative mechanism by which the gene transfer occurred.

There is good reason to suppose that introns in eukaryotic genes have an ancient origin $^{2.3}$, so it seems unlikely that an intronless form of the gene was transferred. The authors propose that the recipient Rhizobiaceae acquired DNA encoding a homologue of the cellular glutamine synthetase (GS) gene, complete with introns, which were subsequently deleted in the absence of any obvious selective advantage to cells possessing two GS genes. However, the alternative explanation that immediately presents itself is that the Rhizobiaceae have acquired a processed gene or pseudogene $e^{4.5}$ of plant GS derived from transcribed RNA. Mediation by a plant virus $^{6}$ can even be considered.

The incorporation of processed genes or pseudogenes into the eukaryotic genome is well documented. Reverse transcriptase-like activity "may be the major cause of movement of mobile DNA elements within the nuclear DNA of perhaps all eukaryotes"2 and, given the evidence of Carlson and Chelm ${ }^{1}$, perhaps also between eukaryotes and prokaryotes.

CSIRO,

A.G. BrownleE

Division of Animal Production,

PO Box 239,

Blacktown, NSW 2148, Australia

1. Carlson, T.A. \& Chelm, B.K. Nature 322, 568-570 (1986)

2. Darnell, J.E. \& Doolittle, W.F. Proc. natn. Acad. Sci U.S.A. 83, 1271-1275(1986)

Senapathy, P. Proc. natn. Acad. Sci. U.S.A. 83, 2133-2137 (1986)

4. Sharp, P. A. Nature 301, 471-472(1983)

5. Baltimore, D. Cell 40, 481-482 (1985).

6. Pfeiffer, P. \& Hohn, T. Cell 33, 781-789 (1983).

\section{Caught in a trap}

SiR-We were pleased to read your report' "Catching atoms in beams of light" on our recent experiment ${ }^{2}$. It contains, however, an unfortunate misconception: specifically, that the essential requirement for success was the suspension of conventional belief as "outlined" in the paper of Pritchard et al. ${ }^{3}$ on the scattering force and the optical Earnshaw theorem. Since, as your report correctly states, our trap is due to the dipole force, the work of Pritchard et al., which contains new trapping proposals based on the scattering or spontaneous force, does not apply to our trap and had no influence on our experimental work. In fact, one of the principal conclusions of our paper is that the trapping results are in excellent agreement with previous existing theoretical and experimental knowledge as developed over the past 16 years, and thus are in no way contrary to "conventional belief".

Pritchard's views of the optical Earnshaw theorem ${ }^{4}$ and scattering force traps, as quoted in your report, involve issues that are quite separate from the above. We feel that his opinion, stating in essence that the optical Earnshaw has somehow been an obstacle to valid laser trapping proposals, is not supported by the published literature.

A. Ashrin

J.E. BJORKHOLM Steven Chu

Electronics Research Laboratory,

AT\&T Bell Laboratories,

Holmdel, New Jersey 07733, USA

1. Nature 322, 403 (1986)

2. Steven Chu, Bjorkholm, J.E. Ashkin, A. \& Cable, A Phys. Rev. Lett. 57, 317 (1986)

Pritchard, D.E., Raab, E.L., Bagnato, V., Wieman, C.E. \& Watts, R.N. Phys. Rev. Lett. 57, 310 (1986)

4. Ashkin, A. \& Gordon, J.P. Opt. Lett. 8, 511 (1983).

\section{Cancer risk assessments in light of Chernobyl}

SiR - The radiation doses received by the population of Europe as a result of the Chernobyl fall-out have stimulated a reappraisal of the biological effects of ionizing radiation at low levels of exposure. Although the International Commission on Radiological Protection (ICRP) argue that an assumption of linearity at low dose is "over-cautious", there is evidence from animal experimentation ${ }^{2}$ and human observation $^{3}$ that high LET (linear energy transfer) radiation becomes more dangerous per unit dose at lower doses and not less dangerous. Whereas these observations are of particular relevance to the excess cases of child leukaemia observed around nuclear facilities in the United Kingdom $^{4-7}$ they also emphasize that ICRP is not as cautious as it might appear. Furthermore, it is difficult to understand why ICRP waited until 1957 before adopting a non-threshold model. Ten years previously, D.G. Catcheside submitted a paper to the MRC Radiation Protection Subcommittee which stated: "All quantitative experiments show that even the smallest dosage of radiation produces a genetic effect, there being no threshold dose below which no genetic effect is induced" $"$.

Table 1 Life-time cancer risk per rem per million persons exposed (assuming linearity)

Authority Year $\quad$ Fatal cancers $\quad$ All cancers

$\begin{array}{llll}\text { ICRP } & 1977 & 125 & \\ \text { UNSCEAR* } & 1977 & 100-440 & 300-700 \\ \text { BEIR III } & 1980 & 167-501 & 260-880 \text { (males) } \\ & & & 550-1,620 \text { (females) }\end{array}$

*Excludes the atomic bomb studies.
The ICRP risk estimates are based mainly on atomic bomb data, even though the dosimetry is known to be unreliable and is now undergoing extensive revision". Table I includes data from a paper by Charles and Lindop ${ }^{10}$, which analyses biological data in UNSCEAR $1977^{11}$ but excludes the atomic bomb studies. The ICRP estimates 125 fatal cancers per rem per million persons exposed, which is less than one-third of the upper estimate offered by UNSCEAR, and one-quarter of the upper estimate offered by the US National Academy of Sciences BEIR III Committee $^{12}$. The risks are more than doubled if cancer incidence, rather than cancer mortality is considered, producing ranges of $260-880$ for males, and $550-$ 1,620 for females ${ }^{13}$. Because public exposure limits around nuclear facilities in the United Kingdom at 0.5 rem per annum are twenty times higher than those allowed in the United States, Germany or Japan, it is difficult for governments relying on the ICRP to claim that their standards of safety are amongst the highest in the world.

Furthermore, the recent MRC mortality study of the UK nuclear workforce demonstrates dose-dependent relationships for exposure to ionizing radiation and excess cases of both cancer and leukaemia three times greater than those predicted by the IRCP "assuming that these risks are spread over a twenty-five year period" ". In fact cancer risk estimates are spread over a forty year period. Therefore the observed increases are five times greater than those predicted by IRCP. On this basis, only deep sea fishing is more dangerous than working in the nuclear industry.

These considerations are also of relevance when calculating the biological impact of the Chernobyl fall-out on the UK population. The population of North Wales, Northern Ireland, Scotland and North-West England will receive 400,000 rems over the next five years ${ }^{15}$. Using a linear non-threshold model, one can expect 50 extra cancer fatalities according to ICRP, 50-176 according to UNSCEAR, and up to 500 fatal and non-fatal cancers (excluding skin cancer), according to BEIR III. When calculating effects in other parts of Europe, it is essential that these different estimates are taken into consideration. It is also apparent that for the second time in its short history, there are major discrepancies between the re- 